\title{
Advances and trends in advanced oxidation processes
}

\author{
Dionissios Mantzavinos ${ }^{1}$ Ioannis Poulios ${ }^{2} \cdot$ Albin Pintar $^{3}$
}

Received: 24 October 2016 / Accepted: 28 October 2016

(C) Springer-Verlag Berlin Heidelberg 2016

This special issue of Environmental Science \& Pollution Research consists of ten papers that had originally been presented as oral or poster communications during the fourth European Conference on Environmental Applications of Advanced Oxidation Processes (EAAOP4, http://www.eaaop4.com) held in Athens, Greece, between 21 and 24 October 2015. This well-established series of events attracts researchers and professionals from all over the world to discuss advances and recent trends in the field of AOPs and environment-related applications. More than 200 participants of the present edition of EAAOP-4 came from 30 countries from different geographical areas including the EU (Austria, Cyprus, France, Germany, Greece, Ireland, Italy, Portugal, Slovenia, Spain, U, etc.), Mediterranean countries (Algeria, Egypt, Israel,

Responsible editor: Philippe Garrigues

Dionissios Mantzavinos

mantzavinos@chemeng.upatras.gr

Ioannis Poulios

poulios@chem.auth.gr

Albin Pintar

albin.pintar@ki.si

1 Department of Chemical Engineering, University of Patras, University Campus, Caratheodory 1, Patras, GR-26504, Greece

2 Department of Chemistry, Laboratory of Physical Chemistry, Aristotle University of ThessalonikiGR, 54124 Thessaloniki, Greece

3 Department for Environmental Sciences and Engineering, National Institute of Chemistry, Hajdrihova 19, SI-1001 Ljubljana, Slovenia
Tunisia, Turkey), as well as Brazil, Colombia, India, Mexico, Russia, Serbia, Switzerland, and the USA. They produced a valuable body of information and knowledge, contained in more than 300 oral and poster contributions. The EAAOP conference is now mature since its first edition in September 2006 (Crete, Greece), which points out the increasing interest of the scientific community on AOPs due to the continuous and unsustainable widespread contamination of the environment, biota, and humans by persistent toxic substances, as well as their implications for health and sustainability. The fifth edition of the conference-EAAOP5-will take place in Prague, Czech Republic, in June 2017 (http://www.eaaop5.com).

The collection included in this special issue covers topics such as process integration (e.g., AOPs coupled either together or with biological processes) for the efficient treatment of agro-industrial effluents, experimental and modeling studies, solar photocatalysis in less conventional reactor setups, environmental chemistry and ecotoxicity of transformation by-products, determination of reactive oxygen species, and testing of materials with enhanced catalytic properties. All these clearly reflect the multi- and inter-disciplinary approach needed to perform research of high calibre in the field of AOPs. Indeed, it is quite rewarding to see the participation of researchers with different backgrounds working at the interface of science and engineering to understand and develop technologies for water, air, and soil remediation.

This issue was only possible with the generous contribution of the authors and the journal's editors and editorial assistants who handled the submissions. We are also indebted to all the reviewers who helped in making this special issue an excellent reference material. 


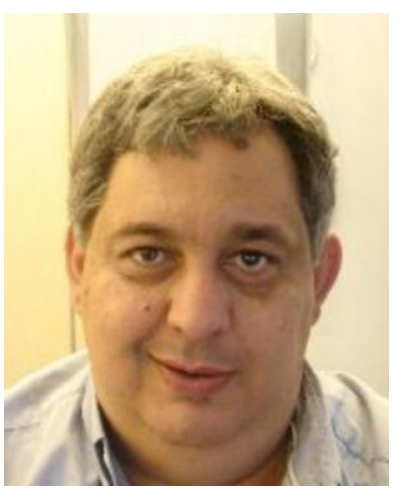

Dionissios Mantzavinos serves as a Professor of wastewater engineering in the Department of Chemical Engineering, University of Patras, Greece. He holds a Diploma (1991) in chemical engineering from Aristotle University of Thessaloniki, Greece, and M.S. (1993) and Ph.D. (1996) degrees in chemical engineering from Imperial College of Science, Technology, and Medicine, UK. After a short spell as a Research Fellow and Lecturer at Edinburgh and Leeds Universities, respectively, he returned home to take up an academic position as an Assistant, Associate, and Full Professor in the Department of Environmental Engineering at the Technical University of Crete before his move to University of Patras. His research interests focus on water/ wastewater treatment by physical, chemical, and biological processes, as well as on environmental catalysis. Particular emphasis is given to advanced oxidation technologies including heterogeneous and homogeneous photocatalysis, electrochemical oxidation, ultrasound radiation, wet air oxidation, ozonation, and various combinations of the above. Mantzavinos is involved in several academic and professional bodies and serves as executive editor to the Journal of Chemical Technology \& Biotechnology and associate editor to Water Research.

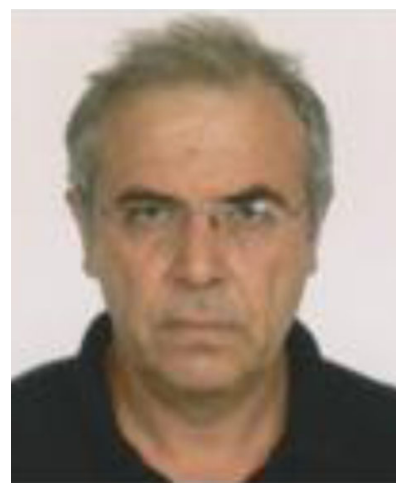

Ioannis Poulios was born in Serres, Greece in 1954. He received his Diploma in Chemical Engineering from the Technical University of Graz, Austria in 1979 and his Dr. Tech. degree from the same University in 1982. (Ph.D. thesis title: $\mathrm{ZnO}$ photoeffects under galvanostatic and potentiostatic conditions). $\mathrm{He}$ is currently Professor in the Laboratory of Physical Chemistry at the Department of Chemistry at the Aristotle University of Thessaloniki. His research interests include Photoelectrochemistry of Semiconductors, Photoelectrochemical Solar Cells, Heterogeneous and Homogeneous Photocatalysis, Solar Detoxification and Disinfection of Wastewaters, Drinking Water and Air. His most recent research efforts are focused on hydrogen production by using photoelectrochemical/photosynthetic processes. He was organizer of four International Conferences on the Environmental Applications of Advanced Oxidation Processes, he is acting as a referee for most of the journals in the area of Environmental Sciences, and was guest editor of several Special Issues. Prof. I. Poulios was in the period 2009-2011 director of the Division Physical, Analytical and Environmental Chemistry of the Chemistry Department and currently director of the Physical Chemistry Laboratory. Full current CV with publication lists can be found at http://photocatalysisgroup.web.auth.gr

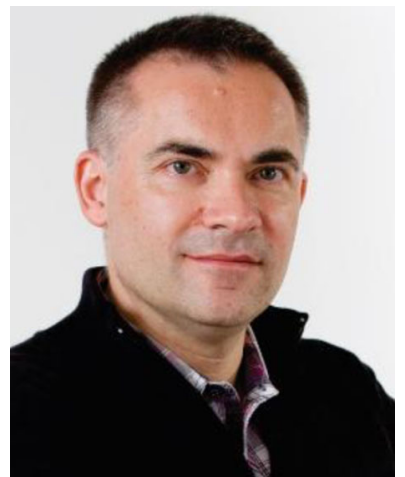

Albin Pintar is head of the Department for Environmental Sciences and Engineering at the National Institute of Chemistry, Ljubljana, Slovenia, and associate professor of chemical engineering at the Faculty of Chemistry and Chemical Technology, University of Ljubljana. He obtained his M.Sc. and Ph.D. degrees from the University of Ljubljana in 1992 and 1996, respectively. $\mathrm{He}$ was a visiting scientist at the Tokyo Institute of Technology (Japan) and Research Institute of Catalysis and Environment (Villeurbanne, France). His research interests are in the fields of heterogeneous catalysis, environmental catalysis, kinetics and mechanisms of catalytic reactions in multiphase reactors, wastewater treatment, production of hydrogen rich mixtures from renewables and process intensification. He is the author of over 130 scientific papers and review articles in peer-reviewed journals, and several international patents. In 2011, he received a prestigious national award for significant scientific achievements in chemical engineering. Albin Pintar is president of the Slovenian Catalysis Society and serves as associate editor to RSC Advances. 